\title{
RESPOSTA DE NIVEIS FREÁTICOS DO SISTEMA AQUIFERO BAURU (FORMAÇÃO ADAMANTINA) EM FUNÇÃOO DA PRECIPITAÇÃO E EVAPOTRANSPIRAÇÃO SOB DIFERENTES USOS DA TERRA
}

\author{
WATER TABLE RESPONSE AT BAURU AQUIFER SYSTEM \\ (ADAMANTINA FORMATION) ACCORDING TO PRECIPITATION AND \\ EVAPOTRANSPIRATION UNDER DIFERENT LAND USES
}

\author{
Aira Nava ${ }^{1}$; Rodrigo Lilla Manzione ${ }^{2}$
}

Artigo recebido em: 10/03/2015 e aceito para publicação em: 26/06/2015. DOI: http://dx.doi.org/10.14295/ras.v29i2.28402

\begin{abstract}
Groundwater monitoring is an essential tool for water resources planning since the response of the water table to precipitation events varies according not only to local hydrogeological conditions but also on land use and management. Thus, this study has aim to apply time series models based on groundwater monitoring data for understanding the processes that occur during the hydrological cycle and affect the availability of groundwater resources of the Bauru Aquifer System (BAS), one of the major groundwater sources available in the Médio Paranapanema hydrographical region, São Paulo, Brazil. The result has shown a distinct response between wells located under the same geological formation but with different land uses. It was noted that in the agricultural area the levels were more sensitive to seasonal variations and management practices, denoting local flow cells, while in the forrest conservation area where there is no anthropic perturbations the monitoring data reflect the base flow in direction to the nearest drainage, influenced by the intermediate/regional groundwater flow.
\end{abstract}

Keywords: Time series. Impulse response function. PIRFICT model.

Resumo: O monitoramento das águas subterrâneas é uma ferramenta essencial para o planejamento dos recursos hídricos, tendo em vista que a resposta dos níveis freáticos aos eventos de precipitação varia não só pelas condições hidrogeológicas locais mas também em função do uso, manejo e ocupação da terra. Desta forma, o presente trabalho teve como objetivo a aplicação de modelo baseado em séries temporais a partir de dados de monitoramento de águas subterrâneas para compreensão dos processos que ocorrem durante o ciclo hidrológico e afetam a disponibilidade dos recursos subterrâneos do Sistema Aquífero Bauru (SAB), um dos principais mananciais disponíveis na região do Médio Paranapanema. Os resultados mostraram um comportamento distinto entre os poços localizados sob a mesma formação geológica, mas com usos da terra distintos. Notou-se que em área agrícola os níveis foram mais sensíveis às variações sazonais e às práticas de manejo ali empregadas, denotando células de fluxo local, enquanto que em área de conservação florestal onde não há perturbações antrópicas os dados de monitoramento refletem o escoamento de base em direção à drenagem mais próxima, influenciada principalmente pelo fluxo intermediário/regional das águas subterrâneas.

Palavras-chave: Séries temporais. Função de impulso e resposta. Modelo PIRFICT.

\section{INTRODUÇÃO}

A relação existente entre os corpos hídricos superficiais e as águas subterrâneas é de fundamental importância no entendimento do ciclo hidrológico. Em grande parte do território brasileiro o fluxo de base proveniente dos aquíferos alimenta os rios e os mantêm perenes nos períodos secos. Isso ocorre em $90 \%$ dos rios nacionais, exceção ao que acontece nos terrenos cristalinos do semiárido nordestino, os quais não têm capacidade de regularizar seus rios, tornando-os intermitentes. Para entender essas relações é necessário caracterizar a resposta dos aquíferos em função das variáveis climáticas que influenciam o sistema, das características hidrogeológicas in situ e do uso e ocupação da terra (MANZIONE, 2015).

O monitoramento e a avaliação das condições que este meio natural se encontra são

\footnotetext{
${ }^{1}$ UNESP - FCA, Botucatu. GEPAG - Departamento de Solos (aaira.nv@gmail.com)

${ }^{2}$ UNESP, Ourinhos (rlmanzione@gmail.com)
} 
instrumentos fundamentais para tomada de medidas preventivas e/ou proativas que buscam conciliar a qualidade e a quantidade dos recursos. Tendo em vista as diferentes funções das águas subterrâneas (social, ambiental ou mesmo econômica), um programa de monitoramento que seja eficiente deve envolver o controle da qualidade e quantidade, considerando as inter-relações existentes com as águas superficiais, condições climáticas e usos locais (MESTRINHO, 2008).

Informações sobre a dinâmica dos aquíferos são importantes para balancear os interesses econômicos e ecológicos quanto ao uso do solo e da água (VON ASMUTH e KNOTTERS, 2004). Em hidrologia, a dinâmica dos aquíferos é explicada de diversas formas. No campo das análises de séries temporais, modelos de função de transferência de ruído (transfer-function noise models-TFN) são aplicados para descrever a relação dinâmica entre a precipitação excedente e as alturas de lençol freático (BOX e JENKINS, 1976; HIPEL e McLEOD, 1994; TANKERSLEY e GRAHAM, 1994; VAN GEERA e ZUUR, 1997; FABBRI et al., 2011; PETERSON e WESTERN, 2014).

O sistema transforma séries de observações de entrada (variáveis explicativas) em séries de saída (variável de resposta, no caso alturas de lençol freático). Para alturas de lençol freático, a relação dinâmica entre a precipitação e as alturas do lençol podem também ser descritas por modelos físico-mecanísticos de fluxo. Entretanto, modelos muito menos complexos como os modelos de função de transferência de ruído geralmente obtêm predições tão acuradas quanto modelos físicomecanísticos (KNOTTERS e BIERKENS, 2001).

Assim, o objetivo desse trabalho foi caracterizar a resposta dos níveis freáticos do Sistema Aquífero Bauru (SAB) e entender sua relação com diferentes usos da terra em áreas da Formação Adamantina no município de Assis/SP.

\section{MATERIAIS E MÉTODOS}

\section{1 Área de estudo}

Para esse estudo foram selecionados dois poços de monitoramento, distantes aproximadamente 2.300 metros. Um poço está localizado nos domínios da Estação Ecológica de Assis e outro nos domínios da Agência Paulista de Tecnologia dos Agronegócios (APTA) - Polo Regional Médio Paranapanema, ambos no município de Assis/SP (Figura 1). Esses poços atravessam os sedimentos da Formação Adamantina do Grupo Bauru (Anexo 1).

A Estação Ecológica de Assis (EEcoA) detém uma área de 1.760,64 ha $(17,61$ $\mathrm{km}^{2}$ ), o que representa $10 \%$ das áreas de cerrado protegidas em Unidades de Conservação no Estado de São Paulo. É um local de proteção integral dos recursos naturais que visa à preservação da natureza, onde é permitido apenas o uso indireto dos seus recursos. Tem como objetivo principal a preservação da vegetação de cerrado "lato sensu", possuindo uma amostra significativa das áreas limítrofes da face sul da zona de domínio do bioma. O local, que se destaca por uma condição ecotonal, onde parte de sua extensão é ocupada por vegetação de transição devido ao contato entre o Cerrado e a Mata Atlântica (Floresta Estacional Semidecidual), também possui em seu interior a nascente do principal manancial da região (ribeirão do Cervo). Além disso, dispõe de todas as sub-bacias que abastecem o reservatório da SABESP de Assis, localizadas total ou parcialmente na Zona de Amortecimento da Unidade de Conservação (IF, 2010). O poço em estudo nessa área faz parte da Rede Integrada de Monitoramento das Águas Subterrâneas (RIMAS) operada pelo Serviço Geológico do Brasil (CPRM), coordenadas $22^{\circ}$ $36^{\prime} 22^{\prime \prime}$ ' S e $50^{\circ} 22^{\prime} 38^{\prime \prime}$ O, altitude 544 metros (a.n.m), possui uma profundidade de 60 metros e os dados são coletados com uma frequência horária, utilizando sensor tipo transdutor de pressão, desde 2011. 


\section{Mapa de localização dos poços no município de Assis-SP}

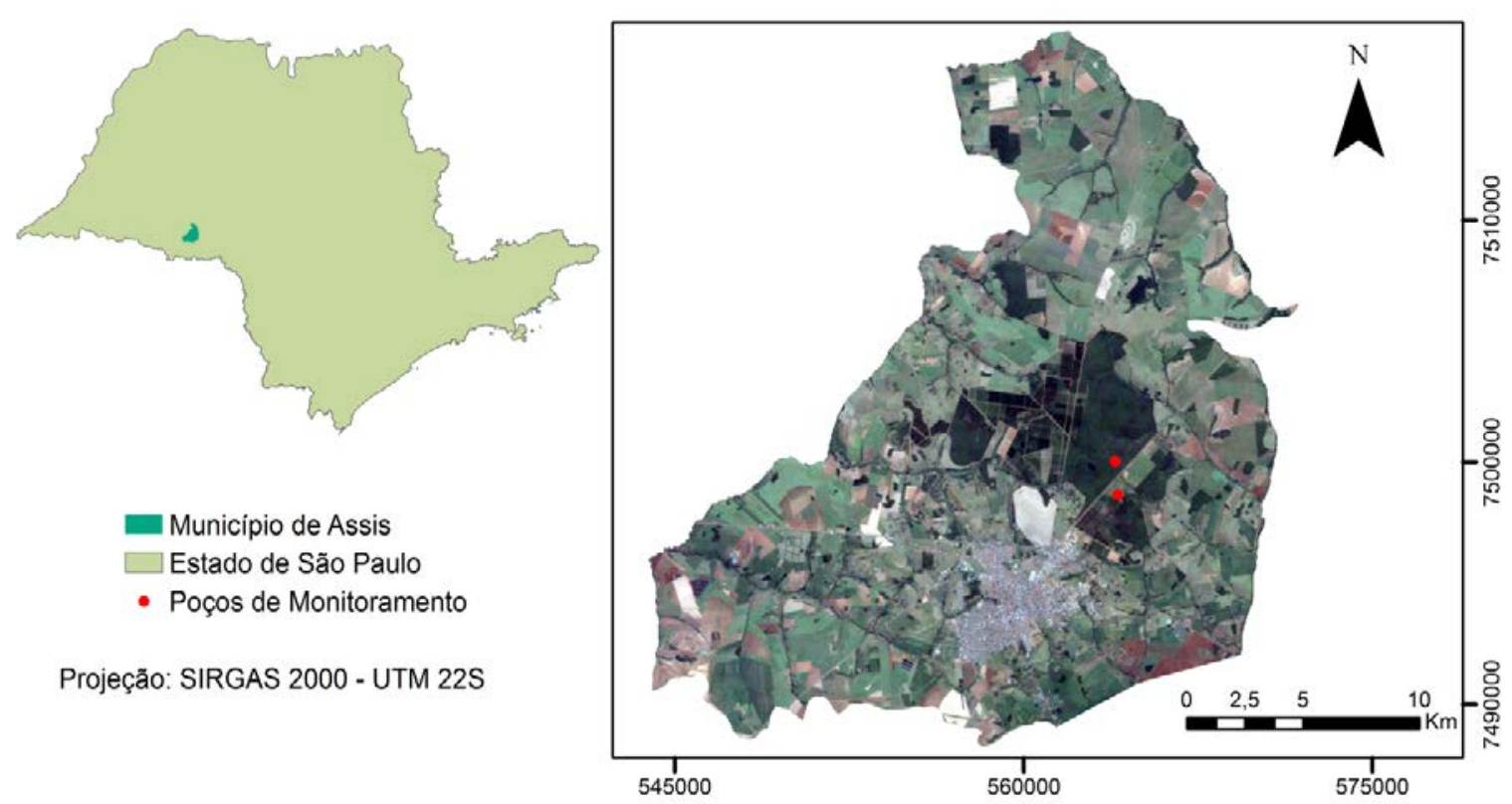

Figura 1 - Localização dos poços no município de Assis, SP

Figure 1 - Wells location in Assis, SP

O poço de monitoramento instalado nas dependências da APTA é resultado do projeto de "Ampliação e modernização da rede de monitoramento hidrológico na região do CBH-MP”. Este projeto foi viabilizado com verbas do Fundo Estadual de Recursos Hídricos (FEHIDRO), e o monitoramento sistemático é realizado pelo Departamento de Águas e Energia Elétrica do Estado de São Paulo (DAEE) desde 2008. O poço está localizado em uma área de cultivo de milho e mandioca em rotação, realizado por meio de técnicas mecanizadas. Encontra-se entre as coordenadas $22^{\circ} 37^{\prime} 04^{\prime \prime}$ 'S e $50^{\circ} 22^{\prime}$ 37' ' O, a uma altitude de 553 metros (a.n.m) e apresenta uma profundidade de 47 metros. Seus dados são coletados com uma frequência quinzenal, utilizando medidor de nível de carretel tipo fita, desde 2008.

\subsection{Série de dados}

A série de observação do nível das águas subterrâneas na EEcoA foram realizadas com frequência diária, cobrindo o período entre julho de 2011 e dezembro de 2013. Na APTA, a série temporal foi registrada com frequência semanal, entre o período de março de 2008 e agosto de 2014.

Também foram utilizadas séries temporais de variáveis climatológicas como precipitação e evapotranspiração, disponibilizados pelo Centro Integrado de Informações Agrometeorológicas (CIIAGRO ONLINE www.ciiagro.sp.gov.br) da estação meteorológica que fica dentro da APTA. Estes dados foram coletados semanalmente e organizados entre os anos de 2002 e 2014. A série de evapotranspiração foi padronizada pelo método Penman- Monteith, reconhecido pela FAO (Food and Agriculture Organization of the United Nations), conforme Allen et al. (1998), levando-se em consideração o coeficiente de cultura.

\subsection{Modelagem proposta}

O comportamento de um sistema linear de entrada e saída pode ser completa- 
mente caracterizado por sua função de impulso e resposta (IR) (ZIEMER et al., 1998; VON ASMUTH et al., 2002). Para isso, utilizou-se o chamado modelo PIRFICT (Predefined Impulse Response Function In Continuous Time). Nesse modelo, são consideradas as séries de observação dos níveis freáticos e as séries de precipitação e evapotranspiração disponíveis, embora o modelo possa trabalhar com múltiplas variáveis de entrada (VON ASMUTH et al., 2008). No caso, o pulso de entrada é transformado em uma série de saída por uma função de transferência em tempo contínuo.

Assumindo-se linearidade no sistema, uma série de alturas de lençol freático é uma transformação de uma série de precipitação excedente, descontando a evapotranspiração potencial. Essa transformação é completamente governada pela função IR. Para o caso de um sistema linear simples, sem perturbações freáticas, que é influenciado somente pela precipitação excedente, o modelo TFN a seguir (escrito como uma convolução integral) pode ser usado para descrever a relação entre alturas de lençol freático e a precipitação excedente (VON ASMUTH et al., 2002):

$$
\begin{aligned}
& h(t)=h^{*}(t)+d+r(t) \\
& h^{*}(t)=\int_{-\infty}^{t} p(\tau) \theta(t-\tau) \partial \tau \\
& r(t)=\int_{-\infty}^{t} \phi(t-\tau) \partial W(\tau)
\end{aligned}
$$

onde: $h(t)$ é a altura de lençol freático observada no tempo $t[T] ; h^{*}(t)$ é a altura de lençol freático predita no tempo $t$ creditado ao excedente de precipitação relativa a $d[L] ; d$ é o nível de $h^{*}(t)$ sem a precipitação ou em outras palavras profundidade do nível freático local, relativo a superfície do solo [L]; $r(t)$ é a série dos resíduos [L]; $p(t)$ é a intensidade do excedente de precipitação no tempo $t[L / T] ; \theta(t)$ é a função de transferência de impulso/resposta (IR) [-], correspondendo a uma função de dis- tribuição Pearson tipo III (PIII df); $\phi(t)$ é a função IR do ruído [-]; e $W(t)$ é um processo de ruído branco contínuo (Wiener) $[L]$, com propriedades $E\{\mathrm{~d} W(t)\}=0, E\left[\{\mathrm{~d} W(t)\}^{2}\right]=\mathrm{d} t$, $E\left[\mathrm{dW}\left(t_{1}\right) \mathrm{d} W\left(t_{2}\right)\right]=0, t_{1} \neq t_{2}$.

A profundidade do nível freático local $d$ é obtido a partir dos dados como se segue:

$d=\frac{\sum_{i=0}^{N} h\left(t_{i}\right)}{N}-\frac{\sum_{i=0}^{N} h^{*}\left(t_{i}\right)}{N}-\frac{\sum_{i=0}^{N} r\left(t_{i}\right)}{N}$

sendo $N$ o número de observações de alturas de lençol freático.

A área e forma da função IR dependem das circunstancias hidrológicas locais de cada poço estudado. A opção por esse tipo de função se dá por sua natureza flexível, ajustandose a uma grande gama de respostas hidrológicas. Assumindo-se linearidade, a componente determinística da dinâmica do lençol freático é completamente descrita pelos momentos da função IR. Nesse caso, os parâmetros podem ser definidos segundo Von Asmuth et al. (2002):

$$
\begin{gathered}
\theta(t)=A \frac{a^{n} t^{n-1} \mathrm{e}^{-a t}}{\Gamma(n)} \\
\phi(t)=\sqrt{2 \alpha \sigma_{r}^{2}} \mathrm{e}^{-\alpha t}
\end{gathered}
$$

onde $A, a, n$, são os parâmetros da curva ajustada, $\Gamma(n)$ é a função Gamma e $\alpha$ controla a taxa de decaimento de $\phi(t)$ e $\sigma_{r}^{2}$ é a variância dos resíduos.

As equações 5 e 6 e seus parâmetros apresentam sentido físico como descrito em Von Asmuth e Knotters (2004). O parâmetro $A$ é relacionado com a resistência à drenagem (a área da função IR é igual a razão entre a altura média do lençol freático e a recarga média). O parâmetro $a$ é determinado pelo coeficiente de armazenamento do solo (porosidade) e $n$ pelo tempo de convecção e dispersão da precipitação pela zona não saturada. As ba- 
ses físicas são explicadas por funções de transferência de uma série de reservatórios lineares (NASH, 1958). O parâmetro $n$ demonstra o número de reservatórios e $a$ é igual ao inverso do coeficiente de reservatório normalmente usado. Von Asmuth e Knotters (2004) chamam atenção para cuidados ao interpretar esses parâmetros da PIII df quanto a seu sentido físico no processo, uma vez que suas bases são empíricas.

O modelo PIRFICT é capaz de lidar com qualquer frequência de dados por ser contínuo no tempo. Além disso, oferece uma vantagem adicional ao calibrar modelos TFN em séries irregulares, comparado a modelos autoregressivos combinados ao filtro de Kalman (KNOTTERS e BIERKENS, 2001), já que o formato da função de transferência não é restrito a um formato exponencial (VON ASMUTH e BIERKENS, 2005).

Originalmente formulado para descrever a variação dos níveis freáticos nos diques holandeses, o modelo PIRFICT demonstrou grande potencial de aplicação à realidade bra- sileira (MANZIONE, 2007). A flexibilidade da função de impulso e resposta que estabelece a relação entre as variáveis climáticas e os níveis freáticos (PIII df) permite ao modelo se ajustar a diferentes sistemas hidrológicos, como nos Cerrados brasileiros (MANZIONE et al., 2010) e em áreas de afloramento do Sistema Aquífero Guarani (MANZIONE et al., 2012).

As análises do modelo PIRFICT foram realizadas utilizando o software Menyanthes (www.menyanthes.nl).

\section{RESULTADOS E DISCUSSÕES}

Utilizando as séries históricas climatológicas de precipitação e evapotranspiração como variáveis de entrada, o modelo PIRFICT foi calibrado com base nas séries temporais dos níveis freáticos do SAB para os poços de monitoramento EEcoA e APTA. Os resultados da calibração do modelo se encontram na Tabela 1.

Tabela 1 - Estatísticas da calibração do modelo PIRFICT às séries temporais observadas.

Table 1 - Statistics of PIRFICT model calibration to the observed time series

\begin{tabular}{cccc}
\hline ID & EVP (\%) & RMSE (m) & RMSI (m) \\
\hline EEcoA & 96,71 & 0,016 & 0,007 \\
APTA & 84,81 & 0,256 & 0,123 \\
\hline
\end{tabular}

EVP: variância explicada pelo modelo; RMSE (m): corresponde à raiz do erro quadrático médio; RMSI (m): erro do modelo entre um instante $t$ em relação à $t-1$.

Por meio dos Percentuais da Variância Explicada pelo modelo (EVP), nota-se que houve um bom ajuste em ambos os poços de monitoramento. Apesar do poço da EEcoA apresentar um melhor ajuste, os valores da Raiz do Erro Quadrático Médio (RMSE) se mostraram baixos em ambos os casos, sendo cerca de $25 \mathrm{~cm}$ na APTA e inferior a $2 \mathrm{~cm}$ na EEcoA. O mesmo se aplica aos valores da Raiz das Inovações Quadráticas Médias (RMSI), que são inferiores a $13 \mathrm{~cm}$ na APTA e a $1 \mathrm{~cm}$ na EEcoA. Este parâmetro corresponde ao erro do modelo entre um instante $t$ em relação à um instante anterior $t-1$.

A diferença dos valores de RMSE e RMSI encontrados nos poços ocorre principalmente pela irregularidade entre as séries observadas. Tem-se que as observações no poço da EEcoA são mais frequentes e possuem um espaçamento regular de tempo entre uma medição e outra. No poço da APTA, há uma menor quantidade de dados e uma frequência de monitoramento menos regular.

Entretanto, outros fatores podem causar estas diferenças estatísticas. Tais como, a proximidade dos níveis freáticos com a super- 
fície, as condições físicas particulares do meio, a vegetação local e o uso do solo (VON ASMUTH, 2012). As Figuras 2 e 3 mostram os ajustes das séries dos níveis freáticos às variáveis de entrada.

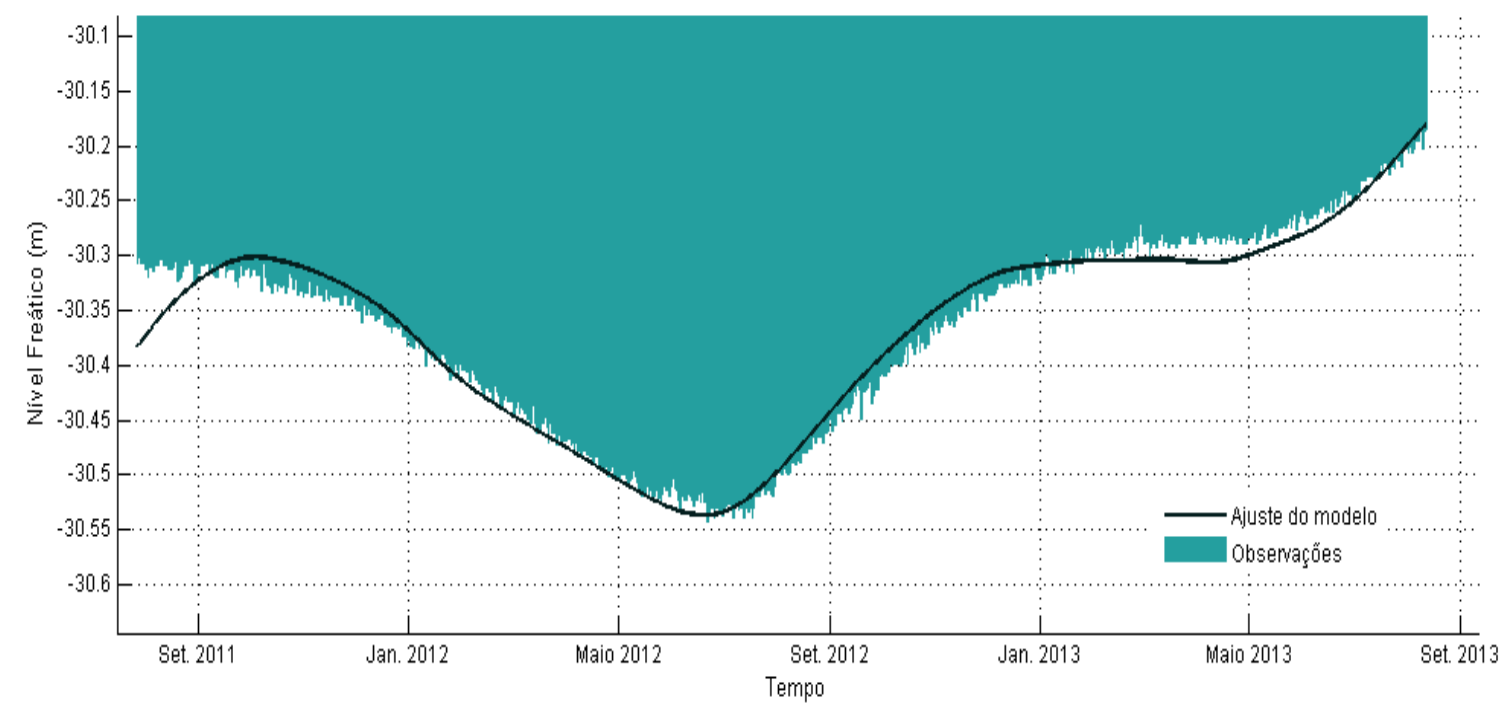

Figura 2 - Comparação entre os dados observados e calibrados pelo modelo PIRFICT para o poço EEcoA Figure 2 - Comparison between the observed data and calibrated data by PIRFICT model for the EEcoA well.

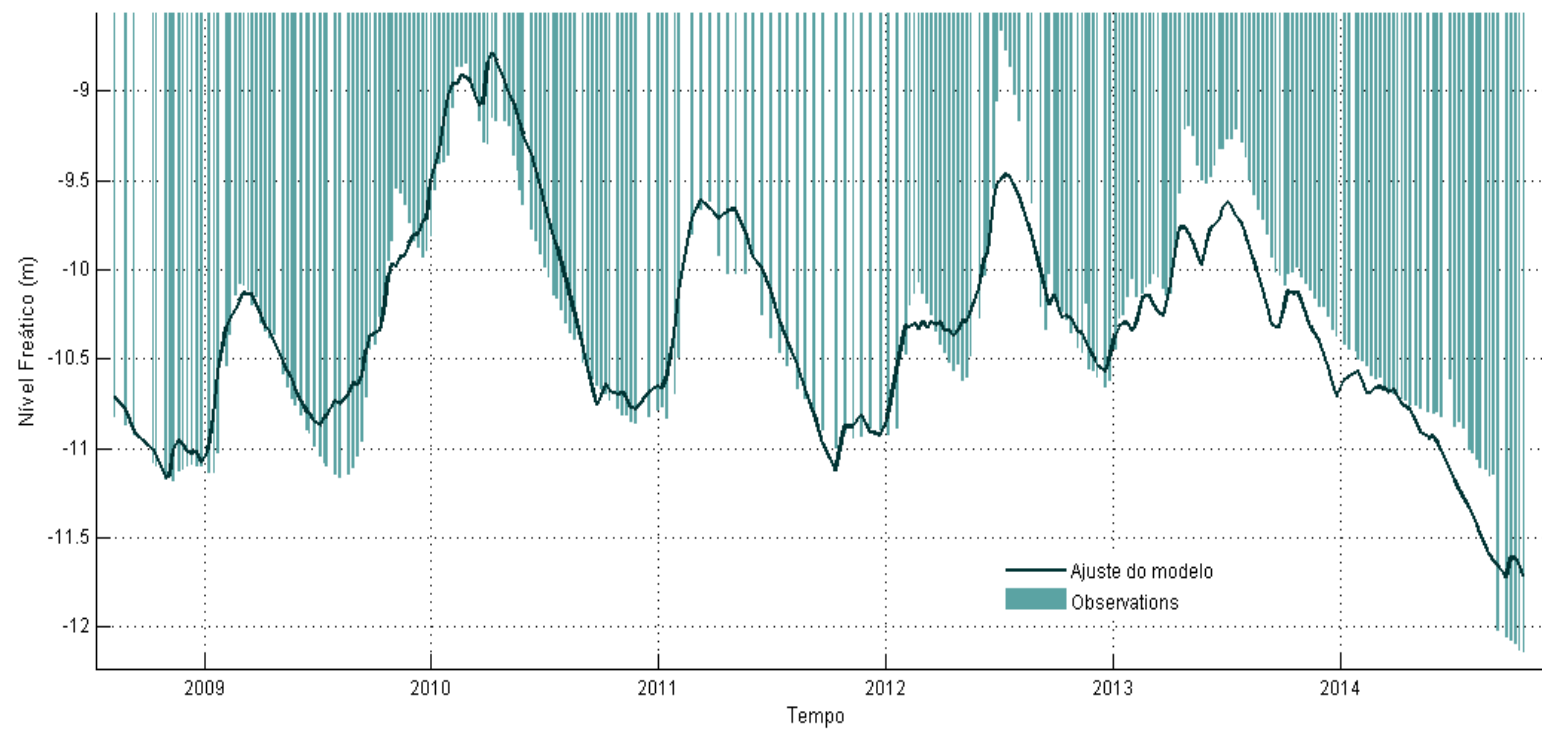

Figura 3 - Comparação entre os dados observados e calibrados pelo modelo PIRFICT para o poço APTA. Figure 3 - Comparison between the observed data and calibrated data by PIRFICT model for the APTA well

Na Figura 2, verifica-se que a linha ajustada acompanha os valores de observação registrados em campo. O bom ajuste ocorre por toda a extensão de tempo, exceto no início da série, em setembro de 2011, no período pós-perfuração do poço.

Já na Figura 3, a linha ajustada não segue tão fielmente os valores das observações, cabendo à função IR capturar a relação dinâmica entre as variáveis de entrada e os níveis freáticos observados e o preenchimento da série estimada.

A calibração também possibilitou estimar os parâmetros de caráter físico que influenciam na oscilação dos níveis freáticos, conforme se observa na Tabela 2. 
Tabela 2- Parâmetros físicos estimados através da calibração do modelo PIRFICT

Table 2 - Physical parameters estimated through PIRFICT model calibration

\begin{tabular}{cccc}
\hline ID & $\boldsymbol{A}$ & $\boldsymbol{a}$ & $\boldsymbol{n}$ \\
\hline EECOA & 7500 & 0,0027 & 2,88 \\
APTA & 1086 & 0,0056 & 1,35 \\
\hline
\end{tabular}

$A=$ resistência à drenagem (dias); $a=$ coeficiente de armazenamento no solo (1/dias); $n=$ tempo de convecção e dispersão (dias)

Desta forma, tem-se que o parâmetro A, por definição, refere-se à resistência à drenagem apresentada pelo meio. Assim, com o aumento dessa resistência em função da espessura do meio poroso, a percolação da água ocorre mais lentamente. Por isso o parâmetro $a$, relacionado à porosidade, se comporta de maneira inversa, sendo maior onde a resposta é mais rápida. Por fim, o parâmetro $n$ se refere ao número de reservatórios lineares (de mesma característica) que se encontram acima do lençol freático. Como explicam Knotters e
Bierkens (2000), um simples reservatório linear (PIII df com $n=1$ ) é igual a um simples modelo físico de coluna de solo unidimensional, descartando fluxo lateral e o funcionamento da zona não-saturada.

As funções de impulso e resposta ajustadas aos dados caracterizaram uma relação dinâmica entre as variáveis explicativas (precipitação e evapotranspiração) e os níveis freáticos. A Figura 4, a seguir, apresenta o momento em que os níveis respondem ao efeito da precipitação.

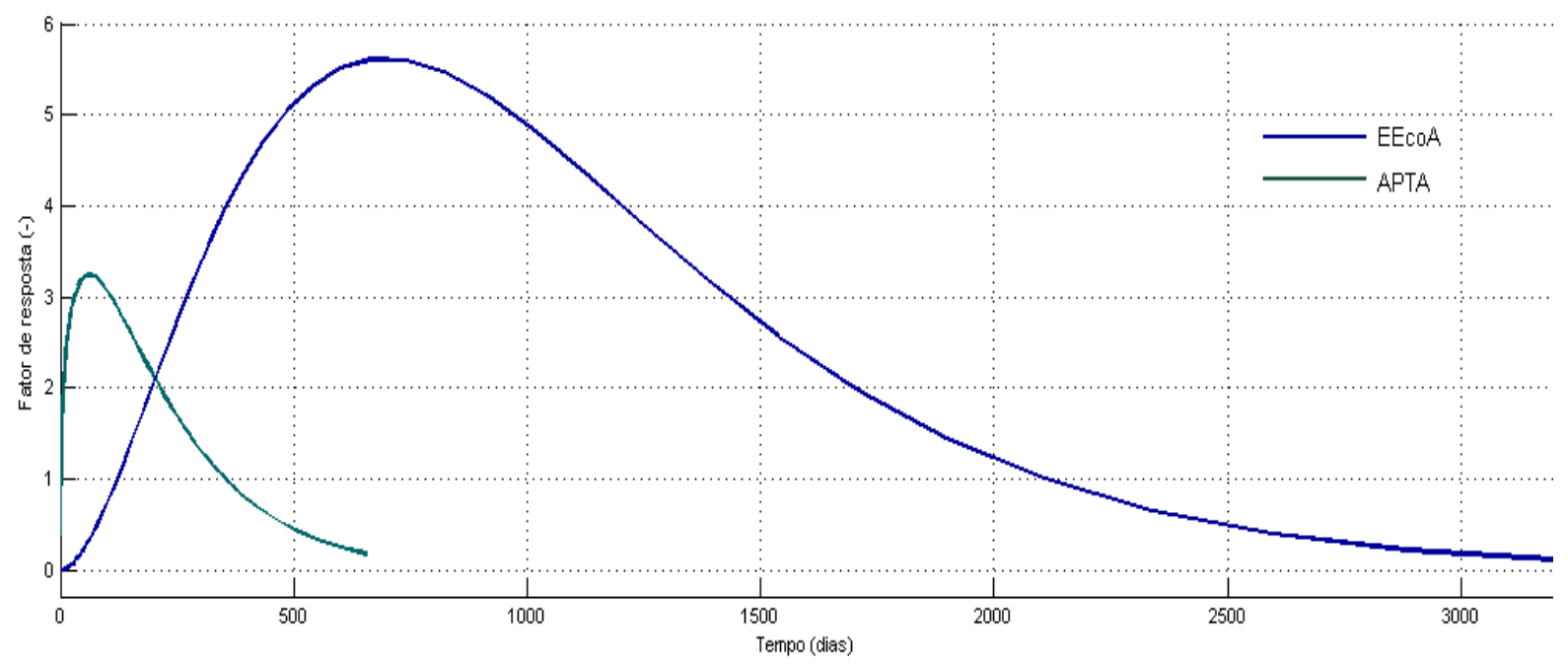

Figura 4 - Comparação entre os gráficos das funções de IR estimadas pelo modelo PIRFICT (azul - EEcoA, verde - APTA)

Figure 4 - Comparison between the IR functions graphs estimated by PIRFICT model (blue - EEcoA, green APTA)

Conforme o tipo de terreno e sua distribuição da paisagem, as águas subterrâneas interagirão de maneiras singulares (WINTER et al., 1998). Os caminhos da água subterrânea variam muito em comprimento, profundidade e tempo de trânsito, a partir dos pontos de recarga para pontos de descarga no sistema de águas subterrâneas. Os níveis freáticos do poço de monitoramento da EEcoA (linha azul) são mais profundos quando comparados aos 
da APTA (linha verde), apresentando uma resposta mais demorada aos estímulos da precipitação. Isto pode ser observado, também, através das áreas encontradas nos gráficos das funções de IR calculadas pelo modelo (Figura 2). Tais áreas representam a zona não saturada pela qual a água deve percolar até gerar algum impacto nos níveis freáticos. Assim, nota-se que a EEcoA possui uma zona superficial não saturada maior que a encontrada na APTA, bem como uma resposta mais tardia aos estímulos.

As curvas ajustadas também mostram que as perturbações nos níveis freáticos são mais frequentes no poço de monitoramento da APTA. Na EEcoA, com base no fator de resposta, o impulso gerado é menos intenso e a perturbação causada que exerce influência na série se dissipa em um maior período de tempo. A oscilação do poço da EEcoA é relativamente pequena, com uma diferença de 17 cm entre o nível médio mais baixo e o nível médio mais alto, enquanto que na APTA essa diferença foi de $89 \mathrm{~cm}$. Nesses casos, a influência da vegetação presente nas áreas de cada poço pode ser verificada. Onde existem cultivos agrícolas, o uso do solo muda com uma frequência alta, de 3 a 4 meses, em virtude do ciclo das culturas ali desenvolvidas. Aliado ao preparo do solo, isso faz com que a água não seja apenas consumida pelas plantas, mas também percole e exerça influência na dinâmica dos níveis. Já na área da EEcoA, a vegetação de cerrado é adaptada à seca, fazendo com que seu desenvolvimento e evapotranspiração diminuam muito na ausência de água e volte a funcionar nos períodos mais úmidos. Assim, com a entrada da precipitação no sistema, o sistema radicular dessas plantas intercepta a maior parte desse conteúdo, fazendo com que pouca água atinja o lençol freático e exerça influência sobre ele. Conclui-se, desta forma, que o comportamento do lençol na área da APTA é muito mais sensível às variações sazonais e às práticas agrícolas ali desenvolvidas, denotando células de fluxo local, enquanto que na EEcoA os dados de monitoramento refletem o escoamento de base para a drenagem mais próxima, influenciada principalmente pelo fluxo intermediário/regional das águas subterrâneas.

É importante ressaltar que quanto maior o parâmetro $n$, maior a influência da zona não saturada na dispersão da água no solo. Assim, aumentando este parâmetro, os pulsos gerados pela precipitação e evapotranspiração diminuem, causando um maior o atraso no tempo de resposta (VON ASMUTH, 2012). Tais informações são compatíveis com os dados ajustados pelo modelo, uma vez que na EEcoA são encontradas três composições diferentes $(n=2,88)$, enquanto que na APTA apenas uma $(n=1,32)$. VON ASMUTH (2012) alerta que para casos onde $n \neq 1$, o parâmetro $a$ referente à porosidade ou coeficiente de armazenamento não pode ser determinado exatamente.

\section{CONCLUSÕES}

O modelo PIRFICT permitiu ajustar, de forma satisfatória, as oscilações dos níveis freáticos em função da precipitação e da evapotranspiração de cada área, caracterizando a relação dinâmica de impulso e resposta entre as variáveis de entrada e os níveis observados. Os valores estatísticos estimados garantiram confiabilidade às análises dos parâmetros físicos calibrados, importantes para a compreensão da dinâmica do aquífero.

Os resultados mostraram um comportamento distinto entre os poços estudados, sendo que o atraso no tempo calculado para que ocorra alguma perturbação no lençol freático do poço da EEcoA e a baixa frequência de impulsos gerados na série, remetem à possibilidade de que a recarga não ocorra diretamente no local, mas que suas oscilações estejam relacionadas a fluxos intermediários a regionais, frutos da dispersão/percolação da água em áreas adjacentes.

Desta forma, para que seja possível realizar uma gestão adequada das águas subterrâneas em áreas nos domínios do Sistema Aquífero Bauru, em consonância com o uso da terra e com a demanda por água das atividades nelas desenvolvidas, é necessário que sejam realizados estudos e monitoramentos contínuos para verificar o comportamento dos níveis freáticos frente à mudanças na cober- 
Resposta de níveis freáticos do sistema Aquífero Bauru (formação adamantina) em função da precipitação e evapotranspiração sob diferentes usos da terra

tura do solo e alterações nos padrões climáticos.

\section{AGRADECIMENTOS}

Os autores agradecem ao Instituto Florestal do Estado de São Paulo e à equipe de monitoramento hidrológico das Unidades de
Assis, pelo apoio nas atividades de campo do projeto (Processo SMA n. 260108 006.514/2010). Agradecem também à CPRM (Serviço Geológico do Brasil), ao DAEE (Departamento de águas e Energia Elétrica do Estado de São Paulo) e à APTA - Polo Regional Médio Paranapanema pelo compartilhamento dos dados.

\section{REFERÊNCIAS}

ALLEN R. G. et al. Crop evapotranspiration - Guidelines for computing crop water requirements. Roma: Food And Agriculture Organization Of The United Nations, 1998. 308 p. (FAO Irrigation and drainage paper 56).

BOX, G. E. P.; JENKINS, G. M. Time series analysis: forecasting and control. 2.ed. San Francisco: HoldenDay, 1976. 575p.

CPTI - Cooperativa de Servicos, Pesquisas Tecnologicas e Industriais. Diagnóstico da situação dos Recursos Hídricos da UGRH 17 - Médio - Paranapanema: Relatório Zero. São Paulo, 1999. 1 CD-ROM.

FABBRI, P.; GAETAN, C.; ZANGHERI, P. Transfer function-noise modelling of an aquifer system in NE Italy. Hydrolological Processes. v. 25, n. 2 p. 194-206, 2011.

IF (Instituto Federal). Plano de Manejo: Estação Ecológica de Assis (SP). São Paulo: IF, 2010. 171 p.

HIPEL, K. W.; MCLEOD, A. I. Time series modelling of water resources and environmental systems. Amsterdam: Elsevier, 1994. 1013p.

KNOTTERS, M.; BIERKENS, M. F. P. Physical basis of time series models for water table depths. Water Resources Research. v. 36, n. 1, p. 181-188, 2000.

KNOTTERS, M.; BIERKENS, M. F. P. Predicting water table depths in space and time using a regionalised time series model. Geoderma, m v. 103, n. 1-2, p. 5177, 2001.

MANZIONE, R.L. Regionalized spatio-temporal modeling of water table depths in the Brazilian Cerrado. São José dos Campos, 2007. 141 p. Tese (Doutorado em Sensoriamento Remoto) - Instituto Nacional de Pesquisas Espaciais (INPE).

MANZIONE, R.L. Águas subterrâneas: conceitos e aplicações sob uma visão multidisciplinar. Jundiaí: Paco Editorial, 2015. 386 p.

MANZIONE, R.L. et al. Transfer function-noise modeling and spatial interpolation to evaluate the risk of extreme (shallow) water-table levels in the Brazilian Cerrados. Hydrogeology Journal, v. 18, n. 8, p. 1927-1937, 2010.

MANZIONE, R. L. et al. Stochastic simulation of timeseries models combined with geostatistics to predict water-table scenarios in a Guarani Aquifer System outcrop area, Brazil. Hydrogeology Journal, v. 20, n. 7, p. 1239-1249, 2012.

MESTRINHO, S. S. P. Monitoramento em água subterrânea. In: FEITOSA, F. A. C.; MANOEL FILHO, J.; FEITOSA, E. C.; DEMETRIO, J. G. A. Hidrogeologia: Conceitos e aplicações. Rio de Janeiro: CPRM/LABHID, 2008. p. 673-686.

NASH, J. E. Determining runoff from rainfall. Proceedings of Institute of Civil Engineer, v. 10, p. 163-184, 1958.

PETERSON, T. J; WESTERN, A. W. Nonlinear timeseries modeling of unconfined groundwater head. Water Resources Research, v. 50, n. 10, p. 8330-8355, 2014. doi:10.1002/2013WR014800.

SOLDERA, B. C.; MANZIONE, R. L. Modelagem de níveis freáticos no Sistema Aquífero Bauru como ferramenta na gestão de recursos hídricos subterrâneos. Ciência geográfica, m v. 16, n. 1, p. 54-61, 2012.

TANKERSLEY, C. D.; GRAHAM, W. D. Development of an optimal control system for maintaining minimum groundwater levels. Water Resources Research, v. 30, n. 11, p. 3171-3181, 1994.

VAN GEERA, F. C.; ZUUR, A. F. An extension of Box-Jenkins transfer/noise models for spatial interpolation of groundwater head series. Journal of Hydrology, m v. 192, n. 1-4, p. 65-80, 1997. 
VON ASMUTH J. R. Groundwater system identification through time series analysis. 2012. 221p. PhD Thesis. TU Delft.

VON ASMUTH, J. R; BIERKENS, M. F. P.; MAAS, C. Transfer function noise modelling in continuous time using predefined impulse response functions. Water Resources Research, v. 38, n. 12, p. 23.1-23. 2002.

VON ASMUTH, J. R.; KNOTTERS, M. Characterising groundwater dynamics based on a system identification approach. Journal of Hydrology, v. 296, n. 1-4, p.118-34, 2004.

VON ASMUTH, J. R.; BIERKENS, M. F. P. Modelling irregularly spaced residual series as a continuous stochastic process. Water Resources Research, v 41, n.
12, p. W12404, 2005.

VON ASMUTH, J. R.; MAAS, K.; BAKKER, M.; PETERSEN, J. Modeling time series of ground water head fluctuation subjected to multiple stresses. Ground water, v. 46, n.1, p. 30-40, 2008.

WINTER T. C.; HARVEY, J. W.; FRANKE, O. L.; ALLEY, W. M. Groundwater and surface water - a single resource. Denver: United States Geological Survey (USGS), Circular 1139, 1989. 79p.

ZIEMER, R. E.; TRANTER, W. H.; FANNIN, D. R. Signals and systems: continuous and discrete. Upper Saddle River: Prentice-Hall, 1998. 622p. 
Resposta de níveis freáticos do sistema Aquífero Bauru (formação adamantina) em função da precipitação e evapotranspiração sob diferentes usos da terra

ANEXO 1 - Mapa geológico do município de Assis - SP. Geological map of Assis, SP.

Fonte: CPTI, 1999

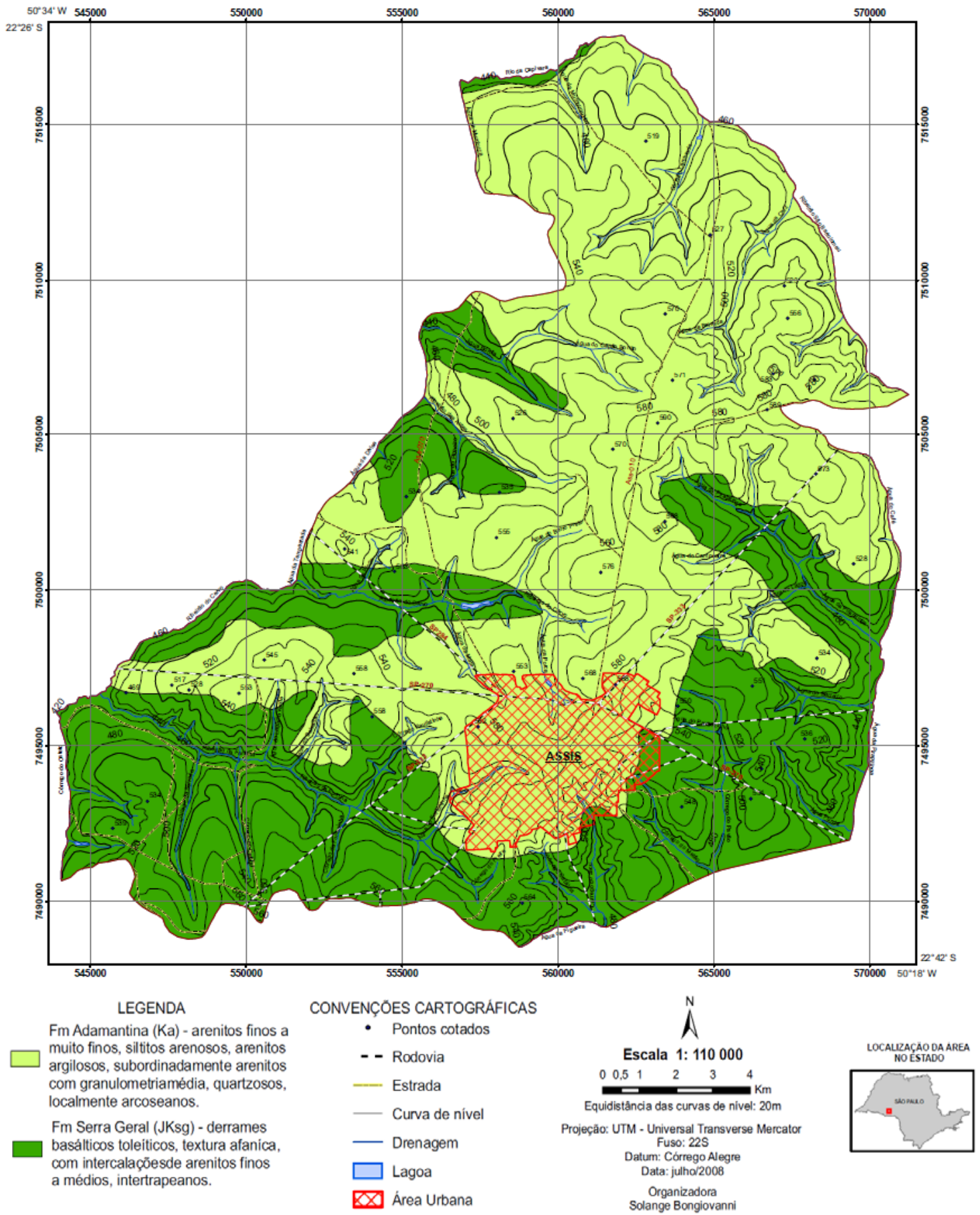

